\title{
Clinical Research and Strategies to Strengthening it
}

\author{
Emilia Campos de Carvalho
}

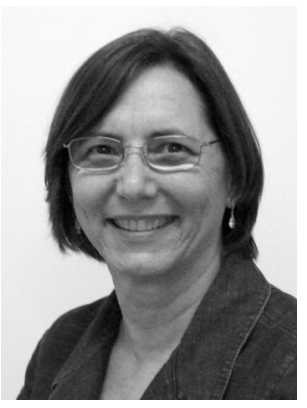

Clinical research, understood as the process of scientific research involving human beings, enables clinical researchers to obtain new scientific knowledge about drugs, procedures or approaches related to problems that affect human health. It is, therefore, a focus of interest for nursing, considering its purpose and the scope of actions professionals can perform in clinical research.

However, determining designs appropriate to research goals and disseminating research steps and findings clearly, concisely and with their essential elements, has been a challenge for researchers, including those in nursing.

Different designs are applied for different purposes. Randomized clinical trials, for example, have been considered the most accurate related to intervention studies. However, other types of studies, such as observational ones, can be more appropriate in certain clinical, ethical or infrastructural situations ${ }^{(1)}$.

Some instruments (guides/lists) have been made available to be used as strategies to lead the process of constructing research projects, mostly for obtaining funds from funding agencies, or for the development of manuscripts to be submitted for the dissemination of research results.

Among the strategies that guide the description of projects or their reports the following are highlighted: the Consolidated Standards of Reporting Trials (CONSORT) ${ }^{(2)}$, containing 22 items that guide the description of randomized clinical trials; Strengthening the Reporting of Observational Studies in Epidemiology (STROBE) ${ }^{(3)}$, which provides information on observational studies such as cohort studies, case control and cross-sectional studies; and the Standards for Reporting of Diagnostic Accuracy (STARD) ${ }^{(4)}$, which can be useful in studies of nursing diagnoses.

In addition to being recommended by some publishers to increase the quality of publications in health journals, these strategies, even under some criticism regarding their use, are useful in developing projects and to new researchers. An example is the set of guidelines disclosed in the area for systematic reviews and meta-analysis studies(3). 


\section{References}

1. Malta M, Cardoso LO, Bastos FI, Magnanini MMF, Silva CMFP. Iniciativa Strobe: subsídios para a comunicação de estudos observacionais. Rev Saúde Pública. 2010; 44(3):559-65.

2. Moher D, Schulz KF, Altman D, CONSORT Group (Consolidated Standards of Reporting Trials). The CONSORT statement: revised recommendations for improving the quality of reports of parallel-group randomized trials. JAMA. 2001;285(15):1987-91. Comment in: JAMA. 2001;285(15):2006-7. JAMA. 2001;286(13):1577-8.

3. von Elm E, Altman DG, Egger M, Pocock SJ, Gotzsche PC, Vanderbroucke JP, et al. Strengthening the Reporting of Observational Studies in Epidemiology (STROBE) statement: guidelines for reporting observational studies. BMJ. 2007;335(7624):806-8.

4. Bossuyt PM, Reitsma JB, Bruns DE, Gatsonis CA, Glasziou PP, Irwig LM, et al. Towards complete and accurate reporting of studies of diagnostic accuracy: the START initiative. Standards for Reporting of Diagnostic Accuracy. Clin Chem. 2003;49(1):1-6.

Emilia Campos de Carvalho is member of the Editorial Board of the Latin American Journal of Nursing, and Full Professor of the University of São Paulo at Ribeirão Preto College of Nursing, WHO Collaborating Centre for Nursing Research Development, Brazil, e-mail: ecdcava@usp.br. 